\title{
Prévention et prise en charge des enfants nés de mères infectées par le VIH au Centre de Santé de Référence (CSRéf) de la commune IV du district de Bamako.
}

\section{Prevention and assumption of responsibility of the children born mothers infected by the VIH with the Center of Health of Reference (CSRéf) of commune IV of the district of Bamako.}

\author{
Nouhoum Telly ${ }^{1,8}$, Benoit Tolofoudié ${ }^{1}$, Mama Sy Konaké2, Mariam Sylla ${ }^{3}$, Mama D Sidibé4, Oumar Sangho ${ }^{5,6}$, Fatou Diawara ${ }^{5,7}$ \\ Sory Traoré ${ }^{8}$, Seydou Doumbia'. \\ ${ }^{1}$ Faculté de Médecine et d'Odontostomatologie, Bamako, Mali \\ ${ }^{2}$ Centre de Santé de Référence de la Commune CIV du district de Bamako \\ ${ }^{3}$ Centre Hospitalo-Universitaire de l'Hôpital Gabriel Touré, Service de Pédiatrie, Bamako, Mali \\ ${ }^{4}$ Centre Hospitalo-Universitaire d'Odonto-Stomatologie, Bamako, Mali \\ ${ }^{5}$ Faculté de Pharmacie, Bamako, Mali \\ ${ }^{6}$ Agence Nationale de Télésanté et d'Informatique Médicale (ANTIM), Bamako, Mali \\ ${ }^{7}$ Centre de recherche d'études et de documentation pour la survie de l'enfant (CREDOS) Bamako, Mali. \\ ${ }^{8}$ Cellule du comité Sectoriel de Lutte contre le Sida, Ministère de la Santé et de l'Hygiène Publique, Bamako, Mali
}

\section{Résumé:}

La transmission de la mère à l'enfant est la principale cause d'infection chez l'enfant, elle peut survenir au cours de la grossesse, de l'accouchement ou de l'allaitement maternel. Dans les pays développés le risque de transmission de la mère à l'enfant a été réduit à moins de $1 \%$ grâce à la large diffusion de l'administration du traitement antirétroviral (TARV) chez les femmes enceintes et chez les enfants exposés au VIH. La présente étude a pour but de décrire la qualité de la PTME au CSRéf de la Commune IV du district de Bamako.

Nous avons mené une étude transversale sur les données de janvier 2013 à décembre 2014 et dont la collecte a été réalisée de Février à Avril 2016, en exploitant tous les dossiers des nourrissons nés de mères séropositives ainsi que ceux de leurs mères. Les données ont été analysées par SPSS version 16. La protection des informations collectées sur les sujets de l'étude a été garantie.

Dans cette étude $55,6 \%$ des mères étaient non scolarisées, $71,1 \%$ diagnostiquées avant la grossesse avec une charge virale indétectable chez $38,9 \%$. Toutes étaient sous ARV avec $92,2 \%$ de bonne observance. Chez $98,3 \%$ des enfants la PCR1 a été réalisée avant l'âge de 2 mois après la naissance et la PCR2 après 6 mois de naissance chez $90,0 \%$ des enfants, près de $99 \%$ ont reçu l'ARV à la naissance et la même proportion le cotrimoxazole après 4 semaines, mais $91,7 \%$ ont fait une infection néonatale. La PCR1 était positif seulement chez $2,8 \%$. Nous avons enregistré $2,2 \%$ de perdu de vue.

Des efforts doivent être faites pour améliorés les statistiques pour l'atteinte des objectifs de la lutte contre le VIH et le sida.

Mots Clés: Prévention, Prise en charge, PTME, VIH et Sida, CSRéf CIV.

\begin{abstract}
:
Mother-to-child transmission is the leading cause of infection in children, and can occur during pregnancy, delivery or breastfeeding. In developed countries, the risk of mother-to-child transmission has been reduced to less than $1 \%$ through the widespread use of antiretroviral therapy (ART) in pregnant women and children exposed to HIV. The objective of this study is to describe the quality of PMTCT in the CSRef of Commune IV of the Bamako district.
\end{abstract}

We conducted a cross-sectional study on data from January 2013 to December 2014, which was collected from February to April 2016, using all records of infants born to HIV-positive mothers as well as those of their mothers. The data were analyzed by SPSS version 16 . The protection of the information collected on the subjects was guaranteed.

In this study, $55.6 \%$ mothers were out of school, $71.1 \%$ diagnosed before pregnancy with an undetectable viral load in $38.9 \%$. All were on ARV with $92.2 \%$ of adherence. In $98.3 \%$ of children, PCR1 was performed before the age of 2 months after birth and PCR2 after 6 months of birth in $90.0 \%$ of children, almost $99 \%$ received ARV and cotrimoxazole at birth and cotrimoxazole after 4 weeks, but $91.7 \%$ had a neonatal infection. PCR1 was positive only for $2.8 \%$. We lost $2.2 \%$.

Efforts should be made to improve the statistics for achieving the objectives of the fight against HIV and AIDS.

Key Words: Prevention, Supported, PMTCT, HIV and AIDS, CSRéf CIV

\section{Introduction}

En 2016, on estimait à 2,1 millions [1,7 million-2,6 millions] le nombre d'enfants vivant avec le $\mathrm{VIH}$ à l'échelle mondiale avec $88 \%$ vivant en Afrique subsaharienne. Environ $50 \%$ des enfants infectés pendant la période périnatale meurent avant leur deuxième anniversaire s'ils ne reçoivent pas de traitement et seulement $43 \%$ [36 \%-54 \%] des enfants 
vivant avec le VIH avaient accès aux traitements antirétroviraux (1). Ces enfants étaient principalement infectés par voie verticale et $90 \%$ de ces infections avaient eu lieu en Afrique subsaharienne (2). Dans les pays développés le risque de transmission de la mère à l'enfant a été réduit à moins de $1 \%$ grâce à la large diffusion de l'administration du traitement antirétroviral (TARV) chez les femmes enceintes et chez les enfants exposés au VIH (3).

La transmission du VIH de la mère séropositive à son enfant peut avoir lieu pendant la grossesse, l'accouchement et l'allaitement. Le dépistage du VIH de la femme enceinte avant l'accouchement est au cœur de la prise en charge des femmes enceintes pour la réduction de la transmission du VIH de la mère à l'enfant (4). Une des stratégies de prévention de la transmission de la mère à l'enfant du VIH (PTME) consiste à administrer des antirétroviraux, en trithérapie chez la mère séropositive et en monothérapie chez le nouveauné. La transmission de la mère à l'enfant est la principale cause d'infection chez l'enfant, elle peut survenir au cours de la grossesse, au cours' du travail et de l'accouchement ou au cours de l'allaitement maternel. En absence de toute intervention et sans allaitement maternel, le risque de transmission du VIH de la mère à l'enfant est de 15 à 30\% (5). L'accès élargi aux services de PTME a permis de prévenir l'infection à VIH de plus de 670000 enfants au cours de la période 2009-2012 (6).

Le Mali est un pays à épidémie généralisée avec une tendance à la baisse. La séroprévalence nationale est de $1,1 \%$ chez les adultes de 15-49 ans selon les données de la dernière Enquête Démographique et de Santé du Mali (EDSM-V (7).

En fin 2016, le nombre de nourrissons, nés de femmes enceintes séropositive, ayant bénéficié d'un dépistage du VIH dans les 2 mois qui ont suivi leur naissance est de 783 nourrissons pour 6065 femmes séropositives attendues soit un taux de réalisation de 12,91.(8).

Une étude menée par Sangho H et al. de 2005 à 2008 dans 4 sites de PTME du Mali a montré une prévalence globale de 6,8\% (262/3855) (9).

Malgré les efforts fournis, peu d'études ont été menées sur la prévention de la transmission mère enfant du VIH dans le CSRéf de la commune IV d'où l'intérêt de la présente étude qui visait à évaluer à partir des données de routine, l'efficacité des stratégies en cours dans la réduction de la transmission mère-enfant du $\mathrm{VIH}$ au CSRéf de la Commune IV du district de Bamako.

\section{Matériels et méthode}

L'étude s'est déroulée au site PTME du Centre de Santé de Référence de la commune IV du district de Bamako. II s'agissait d'une étude transversale sur les données de Janvier 2013 à Décembre 2014 et dont la collecte données a été réalisée entre Février et Avril 2016. Notre population d'étude était constituée par les enfants nés de mères séropositives au VIH dans le CSRéf de la CIV du district de Bamako au Mali.
Notre échantillonnage était exhaustif prenant en compte tous les enfants nés de mères séropositives entre Janvier 2013 à Décembre 2014.

Taille de l'échantillon :

Nous avons obtenu à l'issu du dépouillement des dossiers, une taille d'échantillon de 180 enfants nés de mères séropositives

\section{Critères d'inclusion}

Etaient inclus dans cette étude, tous les enfants âgés de moins de 18 mois nés de mères séropositives, ayant bénéficié d'au moins un test de diagnostic au VIH soit par la PCR ou par la sérologie au VIH dans le CSRéf de la CIV entre Janvier 2013 à Décembre 2014.

Les données ont été recueillies sur une fiche d'enquête individuelle (questionnaire) pré-testée à partir des dossiers médicaux des patients et le registre de consultation.

Description de la technique de collecte et d'analyse de laboratoire des échantillons recueillis Le Dry Blood Spot aussi appelé Dried Blood Spot ou DBS est un prélèvement de sang total recueilli sur du papier filtre absorbant et séché à la température ambiante.

Après une petite piqûre sur le talon, le gros orteil, ou le bout du doigt avec une lancette rétractable de $2 \mathrm{~mm}$, quelques gouttelettes de sang ont étés recueillies sur un morceau de papier filtre absorbant. Ces prélèvements ont été ensuite placés dans un sac plastique à fermeture hermétique (sac zip-lock) accompagné d'un sachet de déshydratant et une carte indicatrice du taux d'humidité. Tous les échantillons ont été analysés à l'Institut National de Recherche en Santé Publique (INRSP) identifié comme le centre de référence national pour le diagnostic des enfants nés de mères séropositives. Tests virologiques utilisés détectent directement la présence du virus VIH dans les échantillons de sang, contrairement au test sur les anticorps, qui détecte la présence d'anticorps comme indicateur indirect de la présence du virus. A ce titre, il existe deux principaux types de tests (i) les tests antigènes P24 détectent l'une des protéines que l'on trouve dans le $\mathrm{VIH}$ (ii) les tests PCR (réaction de polymérisation en chaîne) permettant de détecter l'ADN ou l'ARN viral.

Le test PCR à ADN détecte la présence du virus dans le sang et est utilisé pour le diagnostic du nourrisson de moins de 18 mois alors que le test PCR à ARN permet de détecter et d'évaluer le taux de virus dans le sang (charge virale)

Les tests rapides de diagnostic du VIH ont pour principe : (i) la détection de la présence d'antigène du virus (ii) la détection de la présence d'anticorps du virus (iii) la détermination de la quantité de virus.

-PCR : Polymerase Chain reaction (PCR) ou réaction de polymérisation en chaine: est une technique de biologie moléculaire qui permet de repérer un fragment d'ADN ou de gêne précis, même présent en quantité infime dans un mélange, puis de l'amplifier exponentiellement. 
La PCR est basée sur la capacité de l'ADN polymérase à synthétiser le brin complémentaire d'un ADN servant de matrice. Pour initier le processus, un segment d'acide nucléique doit s'y associer afin de servir d'amorce. Cette amorce (ou primer), de séquence complémentaire à celle du brin à amplifier, est un oligonucléotide synthétique d'une longueur de 17 à 30 bases. Son association à I'ADN aboutissant ainsi à la synthèse d'un ADN double brin. Une réaction de PCR correspond à la succession d'une trentaine de cycles comportant chacun 3 étapes (i) dénaturation de l'ADN $\left(94-95^{\circ}\right)$ (ii) Hybridation ou annelage des amorces $\left(55-57^{\circ}\right)$ (iii) élongation ou extension des amorces $\left(72^{\circ}\right)(10)$.

\section{Variables étudiées}

L'étude a concerné les antécédents médicaux des mères: la période de diagnostic, la mise sous ARV pendant la grossesse, la charge virale plasmatique. Résultats des deux PCR, la mise sous ARV des enfants infectés, le devenir des enfants ayant bénéficié du diagnostic précoce (perdus de vue, décès, dossier clôturé) et le devenir des mères ont été analysés.

Nous avons procédé à des analyses uni-variées descriptives avec l'élaboration de tableaux de fréquences.

Ce travail a été réalisé sur des données sensibles et confidentielles qui étaient gardées en lieu sûr au niveau de la Maternité. Une autorisation du Médecin Chef et du Responsable de l'unité Gynéco-obstétrique a été obtenue pour y accéder. La confidentialité fut garantie lors de la collecte des données en utilisant des identifiants à la place des noms afin de protéger les informations des sujets de l'étude.

\section{Résultats \\ Caractéristiques des mères séropositives}

Les principaux résultats concernant les mères séropositives de cette étude se résument dans le tableau I. Dans cette étude la majorité des femmes était non scolarisée soit $55,6 \%$. Chez $71,1 \%$ des femmes, le diagnostic a été fait avant la grossesse avec une charge virale indétectable chez $38,9 \%$.

Toutes les femmes dépistées positives pendant la grossesse ont bénéficié d'un traitement ARV. La majorité de nos femmes avait une bonne observance (prise régulière en observant la posologie donnée et les consignes données) au traitement ARV soit 92,2\%. Par ailleurs, $1,1 \%$ des femmes avaient arrêté le traitement ARV après l'accouchement et $2,8 \%$ ont été portées disparues.

Nous avons noté que le Centre a pu dépister près de $61 \%$ des conjoints dont $60,0 \%$ séropositives.

Toutes les femmes de notre étude ont poursuivi le traitement après l'accouchement à l'exception de 2 femmes qui ont arrêté et 5 perdues de vue.

\section{Caractéristiques des enfants nés de mères} séropositives

Le tableau II récapitule les résultats sur les enfants concernant les activités de la PTME au CSRéf de la C IV du district de Bamako. Chez $98,3 \%$ des enfants la PCR1 a été réalisée avant l'âge de 2 mois après la naissance et I la PCR2 après 6 mois de naissance chez $90,0 \%$ des enfants.

La plupart des enfants nés de mères séropositives $(91,7 \%)$ ont fait une infection néonatale. Par contre, près de $99 \%$ des enfants ont reçu l'ARV à la naissance.

Chez 98,9\% d'entre eux, une prophylaxie avec le COTRIMOXAZOLE a été instaurée à 1 mois.

II faut noter que presque tous les enfants ont fait la PCR1 et dans une large proportion soit $97,2 \%$ le résultat était négatif. Plus de $90 \%$ des enfants avaient bénéficié de la PCR2 mais il y avait des problèmes de disponibilité des résultats pour $32,2 \%$ d'eux et 9 enfants ne l'avaient pas fait. La majorité $(87,2 \%)$ des enfants avait un dossier clos (sérologie M18 négative) et 2,8\% des enfants ont été mis sous ARV.

\section{Commentaires et Discussion :}

Notre étude a porté sur les données de 180 mères séropositives ainsi que celles de leurs enfants au CSRéf de la CIV enregistrées entre Janvier 2013 et Décembre 2014.

\section{Caractéristiques des mères : Niveau d'étude}

Dans notre échantillon, plus de la moitié des mères $(55,6 \%)$ n'étaient pas scolarisées. Le taux élevé d'analphabétisme des mères a été retrouvé dans une étude menée par Traoré MK qui obtient 39,6\% (11).

Ces proportions élevées pourraient s'expliquer par le faible taux de scolarisation des filles au Mali.

\section{Moment du dépistage}

Chez la majorité des femmes $(71,1 \%)$, le dépistage du $\mathrm{VIH}$ a été fait avant la grossesse, 21,1\% pendant la grossesse et $8,6 \%$ après l'accouchement. Ce taux élevé de dépistage avant la grossesse pourrait être lié à la qualité de sensibilisation sur le dépistage gratuit et l'obtention de consentement. En Afrique du sud, Technau en 2014 retrouve $12 \%$ avant la grossesse, $53 \%$ pendant la grossesse, $35 \%$ après l'accouchement (12). Une étude réalisée à Abidjan, par Dainguy et al. En 2014 sur 54 couples mères-enfants trouve $44,4 \%$ avant la grossesse et $55,6 \%$ pendant la grossesse (13). Cette différence en faveur du notre serait due à la bonne collaboration entre les services de consultation prénatale et ceux de la PTME d'une part et les différentes sensibilisations d'autres parts.

\section{Prise d'ARV}

Toutes les femmes dépistées positives au VIH pendant la grossesse ont bénéficié d'un traitement ARV. Ouédraogo Yugbaré S $O$ et al. dans leur étude précisent que 46,4\% ont reçu leurs ARV aux environs de la 14ème semaine d'aménorrhée (14).

\section{Statut des conjoints}

Dans notre centre d'étude près de $61 \%$ des conjoints dont $60,0 \%$ séropositives a pu être dépisté.

La méconnaissance de l'infection VIH du conjoint des femmes séronégatives peut être à l'origine de primoinfections en cours de grossesse, elles-mêmes 
responsables d'une partie des transmissions verticales méconnues en période périnatale (15).

\section{Devenir des mères}

Toutes les femmes de notre étude ont poursuivi le traitement après l'accouchement à l'exception de 3,9\% dont 2,8\% de perdues de vue. Selon Michael E. Herce, en dépit de ces gains impressionnants, jusqu'à $15 \%$ et $17 \%$ des clients de l'option B +, respectivement, ne commencent pas l'ARV ou se perdent lors du suivi dans les 6 mois suivant l'initiation de l'ART (16).

\section{Caractéristiques de l'enfant :}

\section{Prise d'ARV à la naissance}

Sur les 180 enfants inclus, 178 soit $98,9 \%$ ont reçu la prophylaxie ARV à la naissance. La majorité de ces enfants était sous bithérapie Zidovudine+Niverapine. Nos résultats sont similaires à ceux d'une étude réalisée au Burkina Faso par Ouédraogo Yugbaré $\mathrm{S} O$ où presque tous les enfants ont reçu des ARV $(97,4 \%)$ dès la naissance (Névirapine sirop) selon le protocole (14).

De nos jours au Mali selon le protocole national de prise en charge, c'est la monothérapie qui est indiquée chez tous les enfants nés de mères séropositives (17).

\section{Prophylaxie cotrimoxazole}

La prophylaxie Cotrimoxazole vise à réduire les épisodes de pathologies associées chez les enfants. Dans notre étude $98,9 \%$ des enfants ont bénéficié d'une prophylaxie Cotrimoxazole. Sangho et al. obtient $88,8 \%$ (9). Cette proportion élevée de façon générale semblerai lié à un bon suivi des agents de santé et le respect des rendezvous par les mères. Notons que cette prophylaxie est une recommandation de l'OMS chez les enfants exposés au $\mathrm{VIH}$ (enfants nés de mères séropositives) dès le 45ème jour de vie.

\section{Suivi biologique}

Résultats de la PCR

Les 180 enfants ont bénéficié de la première PCR1 dont $5(2,8 \%)$ avaient un résultat positif. Parmi ces enfants 171 ont effectués le test de confirmation appelé PCR2 et les 5 enfants avaient toujours un résultat positif, mais 9 enfants n'ont pas effectué la PCR2.

Parmi ceux ayant effectués la PCR2, 58 (32,2\%) n'ont pas reçu leur résultat pour des raisons de rupture des réactifs et d'absence de recherche active pour le leurs fournir. Sur les 109 enfants vivant à 6 semaines de l'étude faite par Ouédraogo S 0, 76,85\% des enfants ont bénéficié d'un dépistage du VIH par PCR entre 6 semaines et 4 mois. Ces PCR étaient positives chez $3,61 \%(14)$.

Par ailleurs, Sangho et al. trouvent $6,8 \%$ de positivité (9) et Okusanya et al. au Nigeria (Northwest) trouvent 4,7\% de résultats de PCR positif (18). Pour l'étude de Dainguy et al. à Abidjan, sur 54 enfants $5,5 \%$ avaient un résultat de PCR positif de l'infection à VIH (10). A Bangui, N'gbale RN et al. sur 329 enfants, trouvent une prévalence de $8,3 \%$ des enfants qui étaient infectés par le VIH a la PCR (19). Cette positivité de la PCR pourrait liées d'une part à l'inobservance de certaines mères au cours de la grossesse, à la détection et mise sous ARV tardive des mères infectées (lors de l'accouchement) et d'autres parts à l'allaitement artificiel.

\section{Sérologie HIV}

Sur les 180 enfants, 37 (15,8\%) n'ont pas effectué la sérologie à 18 mois pour diverses raisons (perdu de vue, suivi continu). Par contre, 176 ont réalisé une sérologie à 18 mois et nous avons enregistré seulement un seul cas de résultat positif soit $0,5 \%$.Ce résultat est inférieur à celui de Sangho et al. qui, sur 117 enfants, obtient 4 cas positifs soit $4,48 \%$ (9)

\section{Devenir de l'enfant}

A la fin de notre étude nous avons enregistré 4 perdus de vue soit 2,2\%, 157 dossiers soit $87,2 \%$ (sérologie de M18 négative) ont été clos. Le suivi continuait pour 14 soit 7,8\%. Cinq (5) enfants ont été mis sous ARV soit une proportion de 2,8\%. Au moment de notre enquête ces 5 enfants étaient sous suivis dans le centre. Ces résultats positifs pourraient s'expliquer par le fait que certaines femmes ignoraient leur statut avant et pendant la grossesse. Au cours de notre étude nous n'avons enregistré aucun décès.

\section{Conclusion:}

Au terme de notre étude sur les 180 enfants nés de mères séropositives du service PTME au niveau du CSRéf, nous pouvons conclure que, les activités de la PTME ont généré des résultats très encourageants. Cependant des efforts doivent être faites pour améliorés les statistiques en tenant compte que la transmission verticale est le principal mode de transmission du VIH chez l'enfant et que son interrompre peut contribuer efficacement à atteindre les objectifs d'élimination de l'épidémie du VIH. II s'agit d'encourager la promotion du dépistage systématique chez toutes les femmes venant en consultation prénatale et assurer une prise en charge correcte selon le protocole en vigueur.

\section{Références:}

1. WHO-HIV-2017.32-fre.pdf [Internet]. [cité 21 juill 2018]. Disponible sur: http://apps.who.int/iris/bitstream/handle/

2. Ciaranello AL, Perez F, Keatinge J, Park J-E, Engelsmann B, Maruva M, et al. What Will It Take to Eliminate Pediatric HIV? Reaching WHO Target Rates of Mother-to-Child HIV Transmission in Zimbabwe: A ModelBased Analysis. PLOS Medicine. 10 janv 2012;9(1):e1001156.

3. Frange $\mathbf{P}$, Blanche $\mathbf{S}$. VIH et transmission mère-enfant. La Presse Médicale. 1 juin 2014;43(6, Part 1):691-7.

4. Kêdoté NM, Brousselle A, Champagne F, Laudy D. [PMTCT in Benin: Is the pregnant women's consent free and informed?], Prévention de la transmission mère-enfant du VIH/sida au Bénin: le consentement des femmes au dépistage est-il libre et éclairé ? Ethique Santé. 1 déc 2011;8, 8(4, 4):173, 173-9.

5. MINISTERE DE LA SANTE/DIRECTION NATIONALE DE LA SANTE. Rapport des activités de prévention de la transmission du VIH de la mère à l'enfant. 2014. 
6. UNAIDS_Global_Report_2013_fr_1.pdf [Internet]. [cité 22 juill 2018]. Disponible sur: http://www.unaids.org/sites/default/files/media_asset/UNAl DS_Global_Report_2013_fr1.pdf

7. Ministère de la santé. Enquête Démographique et de Santé 5eme édition (EDSM-V). 2012

8. Haut Conseil National de Lutte Contre le SIDA. Cadre stratégique national de lutte contre le VIH et le SIDA csn 2017-2021. 2017.

9. Sangho H, Keïta AS, Keïta HD, Sylla M, Dia A, Mint Tayeb M, et al. Suivi des nourrissons nés de mères séropositives au VIH au Mali, Follow-up of children born to HIV seropositive mothers in Mali. Santé Publique. 29 nov 2013;25(5):655-62.

10. Elsevier. Biologie moléculaire et biologie clinique. Paris; 1998.

11. Traoré M K. Caractéristiques des enfants décédés au cours de leur suivi dans le site PTME du service de pédiatrie du CHU Gabriel Touré. Thèse Med, Bamako; 2010. №521.

12. Technau KG, KalkE, Coovadia A, Black V ,Pickerill S and al. Timing of Maternal HIV Testing and Uptake of Prevention of Mother-to-Child Transmission Interventions among Women and Their Infected Infants in Johannesburg, South Africa. J Acquir Immune Defic Syndr. 2014 April 15; 65(5): e170.

13. Dainguy ME, Folquet AM, Kouadio E et al. Vécu des mères ayant bénéficié de la prévention de transmission Mère Enfant PTME dans un centre de référence à Abidjan. Med d'Afr Noire, 2014; 61(2).
14. OuédraogoYugbaré $S \quad 0$, Zagré $N$, Koueta $F$ et al. Efficacité de la prévention de la transmission mère-enfant du Virus de l'Immunodéficience Humaine par le protocole 2010 de l'Organisation Mondiale de la Santé au Centre Médical Saint Camille de Ouagadougou (Burkina Faso). Pan African Medical Journal. 2015; 22:303.7720 (11).

15. Carine Jasseron. Archive ouverte. Prise en charge des femmes enceintes infectées par le VIH en France à l'ère des multithérapies : des recommandations aux pratiques. Santé publique et épidémiologie. Université Paris Sud Paris XI, 2012. Consulté le 03/10/2017 sur : https://tel.archives-ouvertes.fr/tel-00783714.

16. Michael E. Herce M E, Mtande T, Chimbwandira F et all. Supporting Option $\mathrm{B}+$ scale up and strengthening the prevention of mother-tochild transmission cascade in central Malawi: results from a serial cross-sectional study. BMC Infectious Diseases. 2015 ; 15:328 s12879-015 (13).

17. Ministère de la Santé/Politique et protocoles de prise en charge antirétrovirale du VIH et du SIDA. Novembre 2013

18. Okusanya BO, Ashimi AO, Aigere EO, Salawu S. E et al. Scaling up Prevention of Mother to Child Transmission of HIV infection to Primary health centres in Northwest Nigeria. African Journal of Reproductive Health December 2013, Special Edition on HIVIAIDS; 17 4:130-137.

19. Ngbale RN, Komangoya ND, Diemer $\mathrm{H}$ et al. Difficultés de la prévention de la transmission du VIH de la mère à l'enfant dans les maternités au sud du Sahara: cas de la maternité d'hôpital communautaire de Bangui. Med d'Afr Noire, juillet 2013; 60(7).

TABLEAU I: Caracteristiques des mères ayant participé à l'etude au CSREF de la CIV du district de bamako

\begin{tabular}{llcc} 
& & \multicolumn{2}{c}{ Mère séropositives } \\
\cline { 3 - 4 } Caractéristique & Primaire & 63 & 35,0 \\
Niveau d'étude & Secondaire & 17 & 9,4 \\
Diagnostic & Non scolarisée & 100 & 55,6 \\
& Avant la grossesse & 128 & 71,1 \\
& Pendant la grossesse & 38 & 21,1 \\
& Apres l'accouchement & 14 & 7,8 \\
Charge virale (En copie/ml) & $<50$ & 70 & 38,9 \\
& $50-1000$ & 28 & 15,6 \\
& $1000-100000$ & 17 & 9,4 \\
Prise d'ARV pendant la & $>100000$ & 15 & 8,3 \\
grossesse & Non fait & 50 & 27,8 \\
Observance ARV & Oui & 166 & 92,2 \\
& & & \\
Statut du conjoint & Observance correcte & 166 & 92,2 \\
& Observance non correcte & 13 & 7,2 \\
& Non spécifiée & 1 & 0,6 \\
Poursuite du traitement & Séropositif & 108 & 60,0 \\
après accouchement & Séronégatif & 1 & 0,6 \\
& Statut inconnu & 71 & 39,4 \\
& Oui & 173 & 96,1 \\
& Non & 2 & 1,1 \\
& Perdue de vue & 5 & 2,8 \\
\hline
\end{tabular}


Tableau II : Caractéristiques des enfants ayant praticités à l'étude au CSRéf de la CIV du district de Bamako

\begin{tabular}{llcc}
\hline Caractéristiques & & Effectif & $\%$ \\
\hline Age de la PCR1 & $<2$ & 177 & 98,3 \\
Age de la PCR2 & $2-4$ & 3 & 1,7 \\
& $3-6$ & 9 & 5,0 \\
Infection néonatale & Non fait & 162 & 90,0 \\
& Oui & 9 & 5,0 \\
Prophylaxie ARV à la & Non & 15 & 8,3 \\
naissance & Oui & 165 & 91,7 \\
Prophylaxie cotrimoxazole & Non & 178 & 98,9 \\
& Oui & 2 & 1,1 \\
Résultat de la PCR1 & Non & 178 & 98,9 \\
& Positive & 2 & 1,1 \\
Résultat de la PCR2 & Négative & 5 & 2,8 \\
& Positive & 175 & 97,2 \\
& Négative & 5 & 2,8 \\
& Faite mais résultat non & 108 & 60,0 \\
& disponible & 58 & 32,2 \\
Devenir de l'enfant & Non faite & 9 & 5,0 \\
& Suivi continu & 14 & 7,8 \\
& Suivi clos & 157 & 87,2 \\
& Perdu de vue & 5 & 2,2 \\
& Transféré sous ARV & & 2,8 \\
\hline
\end{tabular}




\author{
La version française du PROMIS-29. Validation \\ psychométrique et valeurs de référence en \\ population
}

\section{The French PROMIS-29. Psychometric validation and population reference values}

\section{J.Costeab A.Rouquettebcd J.M. Valderase M. Rosef A. Leplègebg}

In Revue d'Epidemiologie et de santé Publique, Volume 66 Issue 5, September 2018, Pages 317-324

https://doi.org/10.1016/j.respe.2018.05.563Get rights and content

\section{Résumé \\ Enoncé du problème}

Le PROMIS-29 est un nouveau questionnaire générique de santé perçue de 29 items, développé dans le cadre du Patient Reported Outcome Measurement Information System(PROMIS). L'objectif de cette étude était de réaliser la validation psychométrique de la version française du PROMIS-29 et de proposer des valeurs de référence pour la population en France.

Méthodes

Un échantillon de la population générale $(n=1501)$, représentatif pour l'âge, le sexe, l'activité professionnelle,

\begin{abstract}
Background

PROMIS-29 is a new generic standardized questionnaire measuring self-reported health status. It was developed as part of the Patient Reported Outcome Measurement Information System (PROMIS) in the United States. The objective of this study was to carry out the psychometric validation of a French-language version of PROMIS-29 and to establish general population reference values for France.
\end{abstract}

\section{Methods}

Quota sampling was conducted by an independent polling company (Ipsos) to obtain a general population sample $(n=1,501)$ representative with regards to: gender, age, occupation, region, and population density of the place of residence. Data collected included the results of the questionnaires PROMIS-29 and Short Form Health Survey (SF-36), the presence of selected chronic diseases, and socio-demographic information.

\section{Results}

The French PROMIS-29 demonstrated excellent factorial validity, confirming the 7 -factor model of the original PROMIS-29. The use of modern measurement methods indicated that the PROMIS-29 scales satisfy the important characteristics of unidimensionality and, for five of the seven composite scales, invariance across age, educational level and gender. Gender and age specific (10-year intervals) reference values were generated for PROMIS-29 use in France.

Conclusion la région et le type d'habitat a été obtenu par les méthodes des quotas par une société indépendante (Ipsos). Les données recueillies incluaient le PROMIS29, le SF-36, la présence de certaines maladies chroniques et des informations sociodémographiques.

\section{Résultats}

La version française du PROMIS-29 présente une excellente validité factorielle, confirmant la structure à sept facteurs du PROMIS-29 original. L'utilisation des méthodologies de mesure moderne montre que les échelles du PROMIS-29 satisfont les importantes propriétés d'unidimensionalité, et pour cinq sur sept d'entre elles, d'invariance pour l'âge, le niveau d'éducation et le sexe. Des valeurs de référence par sexe et tranche d'âge de 10 ans ont été établies pour l'utilisation du PROMIS-29 en France.

\section{Conclusion}

La version française du PROMIS-29 peut être utilisée pour mesurer la santé perçue en France dans différents types d'études. II faudra cependant évaluer la sensibilité au changement du questionnaire avant de l'utiliser de manière sûre dans des études longitudinales.

The French version of PROMIS-29 is a valid and reliable measure of self-reported health status in the French population. The instrument's sensitivity to change needs to be evaluated before its use in longitudinal studies can be recommended.

Approche par recherche de consensus : un préalable à la construction d'une échelle d'évaluation du risque de constipation des patients hospitalisés destinée à la pratique infirmière

Consensus approach: Prior to the construction of an evaluation scale of risk constipation patients hospitalized for nursing practice

V.BergeraC.GermainbR.LacomèrecC.BaderbO.GerbouindA.Bé nardb

In Revue d'Epidemiologie et de santé Publique, Volume 66, Issue 5, September 2018, Pages 301-309

https://doi.org/10.1016/j.respe.2018.07.002Get rights and content

\section{Résumé}

Position du problème

La constipation est un problème de santé récurrent en hospitalisation. Les causes sont multiples comme l'existence d'habitudes irrégulières, le manque d'exercice, les antécédents médicaux. Certains médicaments constituent également une cause majeure de la difficulté d'élimination intestinale, parmi lesquels les antalgiques forts, les traitements du système nerveux central et de la sphère digestive. Enfin, un régime alimentaire mal équilibré, une carence hydrique sont des 
facteurs aggravants, tout comme l'anxiété, qui sont des éléments importants à identifier par l'infirmière pour une gestion préventive de la constipation. Dans la littérature scientifique, il n'existe pas d'outil facile d'utilisation et validé pour la pratique autonome de l'infirmière pour évaluer ce risque. C'est pourquoi nous avons décidé de développer une échelle d'évaluation du risque de constipation du patient hospitalisé (ERCoPH) pour la pratique infirmière et la gestion préventive de ce risque.

\section{Méthode}

L'approche par recherche de consensus par informateurs-clés proposée par Pineault et Daveluy repose sur cinq étapes qui consistent tout d'abord en une revue de littérature pour identifier les facteurs de risque de la constipation. Puis, il s'agit d'élaborer un questionnaire reprenant les facteurs retenus à la suite de la revue de la littérature et de les soumettre à un panel d'experts préalablement sélectionnés. Enfin, la dernière étape est d'analyser les résultats de l'enquête de consensus. Seuls les facteurs ayant reçu une note supérieure à 6 par au moins $80 \%$ des experts ayant répondu à l'item ont été retenus.

\section{Résultats}

La revue de la littérature a permis de sélectionner 69 facteurs de risques soumis à des experts dans le cadre de cette approche. L'analyse des réponses des 23 experts a permis de retenir 15 facteurs de risques. Le comité scientifique a ajouté huit facteurs du fait de leur importance dans la littérature et a décidé de regrouper certains facteurs relevant de domaines proches.

\section{Conclusion}

Au total, 19 facteurs de risques ont été sélectionnés et classés par grands thèmes (âge, activité physique, traitements médicamenteux, données sociales, alimentation/hydratation, antécédents médicaux et chirurgicaux et les données environnementales). Ces facteurs ont été testés auprès de 300 patients recrutés dans différents milieux cliniques afin d'étudier leurs performances pronostiques sur la survenue d'une constipation.

\section{Background}

Constipation is a frequent issue during hospitalization. Multiple causes such as the existence of irregular habits, lack of exercise as well as medical history have been identified. Drugs such as strong painkillers, central nervous system therapies and treatments of the digestive tract are a major cause of constipation. Additionally, unbalanced diet, fluid deficiency, and anxiety may aggravate constipation. The consideration of all these risk factors being under the responsibility of nurses. The difficulty to take into account such a multifactorial aetiology in nursing practice and the fact that there is no easy to use and validated tool to assess the risk of constipation in current nurse practice has led us to consider the development of a Risk Assessment scale of Constipation in Patient Hospitalized (ERCoPH) to facilitate preventive management of this trouble. We present here the first step of the elaboration of this scale, the identification of risk factors through a consensus approach after a systematic literature review.

\section{Methods}

The key informants consensus-based approach proposed by Pineault and Daveluy is based on five steps: (1) a literature review to identify risk factors for constipation; (2) the elaboration of a questionnaire containing the factors identified in the first step; (3) preselect a panel of experts; (4) submission the questionnaire to the panel; (5) analysis the results of the consensus survey. Only factors that received a rating $>6$ by at least $80 \%$ of the experts were retained.

Results

The systematic literature review identified 69 risk factors submitted to the 23 experts of the panel. Fifteen risk factors were retained after analyzing the answers of the experts. The Scientific Committee added eight risk factors because of their importance in the literature and decided to group together some factors of the same domain.

\section{Conclusion}

A total of 19 risk factors were selected and grouped by major class (age, physical activity, medication, social data, food/hydration, medical and surgical history and environmental data). These factors have been tested among 300 patients enrolled in different clinical settings as part of the construction and validation of ERCOPH.

\section{Survie des patients vivant avec le VIH-1 sous thérapie antirétrovirale au Maroc}

\section{Survival in HIV-1 patients receiving antiretroviral therapy in Morocco}

H. Titou, N. Baba, J. Kasouati, S. Oumakir, R.

Frikh, M. Boui, N. Hjira

In Revue d'Epidemiologie et de santé Publique, Volume 66, Issue 5, September 2018, Pages 311-316

https://doi.org/10.1016/j.respe.2018.07.001Get rights and content

Résumé

Objectif

Étudier les déterminants de la survie chez les patients $\mathrm{VIH}-1$ sous thérapie antirétrovirale.

\section{Matériel et méthode}

Étude rétrospective d'une cohorte de 182 malades vivant avec le $\mathrm{VIH}-1$ mis sous thérapie antirétrovirale dans le service de dermatologie vénérologie à l'hôpital militaire d'instruction Mohamed $\mathrm{V}$ de Rabat au cours de la période allant du 1erjanvier 2006 au 1er janvier 2017. La mort de toute cause pendant la période d'étude était considérée comme le résultat de l'infection par le VIH. Le test du Log-rank a été utilisé pour comparer les courbes de survie en fonction des déterminants étudiés. Le 